\title{
Identification and Assessment of Repeat Drug Overdose Visits at EDs in Virginia
}

\section{Inderbir Sohi, Erin E. Austin, Jonathan Falk}

Virginia Department of Health, Richmond, Virginia, United States

Objective

To identify and assess the characteristics of individuals with repeated emergency department (ED) visits for unintentional opioid overdose, including heroin, and how they differ from individuals with a single overdose ED visit.

\section{Introduction}

The Virginia Department of Health (VDH) utilizes syndromic surveillance ED data to measure morbidity associated with opioid and heroin overdoses among Virginia residents. Understanding which individuals within a population use ED services for repeated drug overdose events may help guide the use of limited resources towards the most effective treatment and prevention efforts.

\section{Methods}

VDH classified syndromic surveillance visits received from 98 EDs (82 hospitals and 16 emergency care centers) between January 2015 and July 2018. An unintentional opioid overdose, which included heroin, was classified based on the chief complaint and/or discharge diagnosis (ICD-9 and ICD-10) using Microsoft SQL Server Management Studio. ED visits were categorized as either a single or a repeat visit, where a repeat visit was defined as two or more separate ED visit records from the same individual. ED visit records were matched to individuals using medical record number. Each match represented a repeat visit for one person. RStudio was used to conduct Pearson's chi-square tests for sex, race, and 10-year age groups among both visit groups and to assess visit frequency among the repeat visit group.

\section{Results}

Between January 2015 and July 2018, 9,869 ED visits for opioid overdose were identified, of which 734 (7.4\%) were repeat visits among 597 individuals occurring among 57 EDs. The proportion of individuals with repeated opioid overdose visits was significantly different compared to the proportion of individuals with a single opioid overdose visit by sex (male $66 \%$ vs. $61 \%$ ) and age group $(20-29$ years $34 \%$ vs $30 \%$ ) ( $<<0.05$ ). No significant difference was found by race. EDs had an average of 10 individuals who had repeated opioid overdose visits, with a range from 1 to 62 individuals. Individuals with repeated opioid overdose visits made on average 2.2 visits to EDs, with a range of 2 to 6 visits. The overdose visit rate among EDs ranged from 0.6 to 51.3 opioid overdoses per 100,000 ED visits, with four EDs having a rate greater than 40 opioid overdose visits per 100,000 ED visits.

\section{Conclusions}

Approximately $7 \%$ of ED visits during the study period for opioid overdose were identified as repeat visits using the medical record number. Individuals with repeated opioid overdose visits differed from those with a single opioid overdose visit with respect to sex and age. Repeated opioid overdose visits were disproportionately higher for males and individuals aged 20-29. Hospital utilization by individuals with repeated opioid overdose visits can provide information on which EDs or communities that may require further attention. Some limitations of this study are that the method utilized to identify individuals may result in an underestimation of repeat visits because limited personally identifying information was used to match visit records, and repeat visits that occurred before and after the study period would not be captured.

\section{Acknowledgement}

I would like to acknowledge Kelly Shaw and Tony Jing for their assistance with the analysis. 